\title{
Implantação do serviço de atenção farmacêutica em uma farmácia comunitária: um estudo exploratório na cidade de São José do Belmonte -PE
}

\author{
Aldemir Severo Matias ${ }^{1}$; Thiago Adolfo Sobreira Miranda ${ }^{2}$
}

\begin{abstract}
Resumo: A Atenção Farmacêutica é uma ação de que traz inúmeros benefícios tanto para a organização como para a população de um município, sendo assim expressa-se que o farmacêutico tem papel fundamental neste processo de farmacoterapia. Esta prática vem sendo muito abordada na atualidade tanto no meio acadêmico como as farmácias e drogarias, visando-se práticas de saúde e promoção de laços de relacionamentos eficazes entre a organização e os clientes diante da satisfação e fidelização dos mesmos. Neste meio, o presente artigo buscou discutir a implantação da Atenção Farmacêutica em um município do interior de Pernambuco, através de um estudo exploratório e de observações diárias, sendo necessário relatar que na empresa escolhida para estudo de campo teve resultados satisfatórios gerando-se uma implantação realista de atenção e assistência farmacêutica diante dos desafios diários das atividades do profissional farmacêutico. Os resultados obtidos permitem demonstrar que a atenção farmacêutica é um importante aliado para maximização as saúde através de ações de promoções de saúde municipal trazendo adequação a utilização dos medicamentos, fidelização do cliente com a organização através de laços de relacionamento e confiança, diminuição de auto-medicação. Conclui-se assim que o farmacêutico contribui bastante para melhoria do relacionamento cliente/empresa através da Atenção Farmacêutica diante do acompanhamento eficaz e monitoramento das posologias corretas de medicamentos, visando não apenas vender remédios e sim promover saúde a população. Diante disso espera-se que o presente estudo possa contribuir para pesquisas futuras na atual temática abordada.
\end{abstract}

Palavras Chaves: Atenção Farmacêutica, Farmácia Comunitária, São José do Belmonte.

\section{Implantation of the pharmaceutical care service in a community pharmacy: an exploratory study in the city of São José of Belmonte -PE}

\begin{abstract}
Pharmaceutical Care is an action that brings innumerable benefits to both the organization and the population of a municipality, so it is expressed that the pharmacist plays a fundamental role in this process of pharmacotherapy. This practice has been widely approached nowadays in the academic world as well as pharmacies and drugstores, aiming at health practices and the promotion of effective relationships between the organization and the clients in the face of their satisfaction and loyalty. In this medium, the present article sought to discuss the implementation of Pharmaceutical Care in a city in the interior of Pernambuco, through an exploratory study and daily observations, and it is necessary to report that in the company chosen for field study had satisfactory results generating a deployment realistic care and pharmaceutical assistance in face of the daily challenges of the activities of the pharmaceutical professional. The results obtained demonstrate that pharmaceutical care is an important ally to maximize health through actions of municipal health promotions
\end{abstract}

\footnotetext{
${ }^{1}$ Graduação em Matemática pela Faculdade de Formação de Professores de Serra Talhada. Acadêmico do Curso de Ciências Farmacêuticas, pela Faculdade de Juazeiro do Norte - FJN, Ceará - Brasil.

Contato: aldemirseveromatias@hotmail.com;

${ }^{2}$ Graduado em Farmácia pela Universidade Federal do Rio Grande do Norte UFRN; Especialista em Ensino de Química e Farmacologia Clínica pela Universidade Regional do Cariri - URCA; Mestre em Bioprospecção Molecular de Produtos Naturais pela URCA; MBA em Gestão de Processos Industriais pela FJN. Docente no curso de Farmácia da FJN.
} 
bringing suitability to the use of medicines, customer loyalty to the organization through ties of relationship and trust, reduction of self- medication. It is concluded that the pharmacist contributes greatly to the improvement of the client / company relationship through Pharmaceutical Care through the effective monitoring and monitoring of correct dosages of medicines, aiming not only to sell medicines but to promote health to the population. Given this, it is expected that the present study may contribute to future research in the current topic.

Key Words: Pharmaceutical Care, Community Pharmacy, São José do Belmonte.

\section{Introdução}

Diante das contextualizações do serviço farmacêutico e das constantes atualizações dos serviços, percebe-se que a atenção farmacêutica surge para tentar agregar satisfação e qualidade na prestação de serviços de saúde. Neste meio entende-se que a qualificação do profissional é um importante ponto de destaque no mercado competitivo que resulta no uso seguro de instruções para aquisição de produtos e a maximização do custo-benefício diante da relação cliente/farmacêutico, além de ressaltar que a dispensação racional de medicamentos diminui a automedicação e melhora o tratamento dos usuários deste serviço diante de um monitoramento eficaz (MINISTÉRIO DA SAÚDE, 2006).

Neste sentido, percebe-se que as farmácias da atualidade têm seu profissional farmacêutico não apenas para fiscalização de alguns serviços, gerando um enfoque na qualidade do serviço prestado aos seus clientes diante da promoção de saúde para garantir o uso correto de medicamentos diante da assistência, prestabilidade e a reeducação farmacoterapica. Melo (2006, p. 15), revela que com a resolução n 585 de 29 de agosto de 2013, o farmacêutico pode vislumbrar alguns cuidados com objetivo de promover aos seus paciente e usuários dos serviços a otimização farmacoterapica, diante da monitoração e acompanhamento das prescrições médicas para garantir uma melhor qualidade de vida das comunidades do município.

Partindo-se destas primeiras informações e da complexibilidade da implantação da Atenção Farmacêutica nas farmácias e drogarias, este artigo tem por objetivo explanar sobre a implantação do serviço de atenção farmacêutica numa farmácia comunitária. Tendo-se para isso a análise do histórico da atenção farmacêutica, a contextualização das normas existentes para 
implantação deste serviço e as dificuldades de implantação, tudo isso para se explanar se os resultados do mesmo podem garantir para fidelização e satisfação do cliente.

Justifica-se a escolha da presente temática, uma vez que a prestação e acompanhamento correto durante um tratamento levam as pessoas a se medicarem corretamente, minimizando os efeitos da automedicação e posteriormente gerando redes de relacionamento da tríade empresa/cliente/farmacêutico. Demonstrando-se assim que a importância da temática se dá diante agregação do serviço, das orientações medicamentosas, da dispensação correta e dos cuidados do farmacêutico durante o decorrer do tratamento ao se avaliar todas as especificidades de casos abordados.

\section{Método}

Classifica-se esta pesquisa como de natureza qualitativa e exploratória, diante da necessidade de obter informações realistas de fácil e de qualidade como destaca Günther (2006), sendo possível também a averiguação a realidade dos casos observados e explorá-los para análise de resultados (GIL, 2007).

Para realização esta pesquisa, teve-se como campo de estudo uma farmácia localizada no município de São José do Belmonte - PE, que atua no ramo farmacêutico varejista sem manipulação de fórmula, com cerca de 17 anos de mercado. Pode-se entender que esta apresenta uma estrutura comercial sólida e clientela fidelizada, que enxergou na instalação da Atenção Farmacêutica um diferencial competitivo não só para satisfazer sua clientela como também para melhorar o serviço prestado diante da melhoria da dispensação e prestação correta de posologias medicamentosas, onde ressalta-se que a assistência farmacêutica foi implantada na empresa a cerca de três meses e já vem mostrando resultados eficazes de tratamentos. Todos os dados expostos a seguir são resultados de observações e análises verídicas de casos acompanhados pelo farmacêutico durante o período de fevereiro a abril de 2018.

A presente pesquisa teve como base a análise do histórico da Atenção Farmacêutica além de a RDC 44/2009, visto que aplicação da Atenção Farmacêutica a ANVISA - Agencia de Vigilância Sanitária regulamentou este serviço através da RDC 44/2009, onde se observa que o farmacêutico até então apenas item burocrático nas farmácias e drogarias passasse a poder prestar atendimento aos clientes tanto dentro do estabelecimento como a domicilio. 


\section{Resultados e Discussões}

a) Visão Geral da organização: dados de adequação a atenção farmacêutica

A organização escolhida para realização desta pesquisa foi à empresa Comercial Farmacêutica São José EPP, situada a Praça Sá Moraes nº. 09, centro, na cidade de São José do Belmonte. Esta presta serviços de dispensação de medicamentos éticos, genéricos e similares, com licenças em dias além de dispensação medicamentos psicotrópicos devidamente armazenados. Observou-se que a prestação do serviço de atenção farmacêutica é recente e foi enxergada diante da necessidade do serviço na população que carecia de informações para evitar a automedicação.

Para realização deste serviço foi necessário a aquisição de uma sala separada dos demais compartimentos do estabelecimento, toda adequada e climatizada para prestação eficiente à atenção e assistência farmacêutica com a logomarca "Atenção Farmacêutica".

\section{b) Desempenho de serviço ofertado: etapas para fidelização do cliente}

Especifica-se que a oferta deste novo serviço começou no mês de fevereiro do presente ano, com a introdução inicial de aferimento de pressão arterial, teste de glicemia, e prestação de informações aos usuários destes serviços além de acompanhamento de alguns casos através de carteirinha personalizadas.

Nesta perspectiva, tem-se que se observar além da estrutura física implantada na empresa em questão o perfil dos clientes da organização e dos possíveis usuários do serviço da atenção farmacêutica, que pode ser expressa nos gráficos abaixo. 
Gráfico 1: Sexo dos clientes do estabelecimento Fonte: Autor

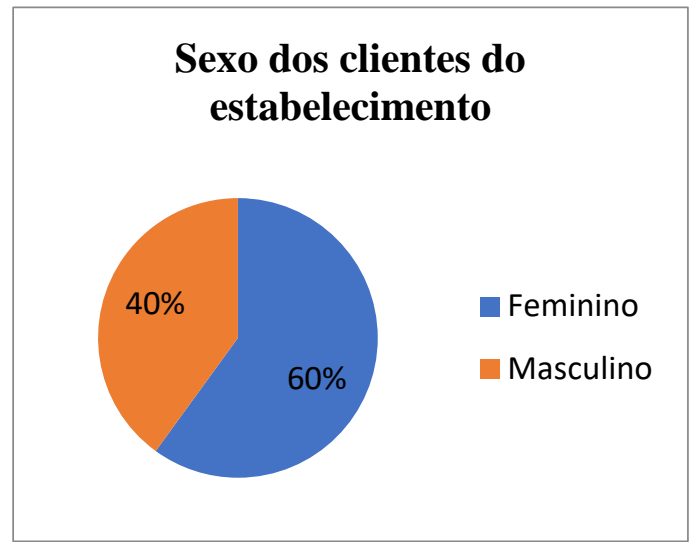

Gráfico 2: Grau de Escolaridade Fonte: Autor.

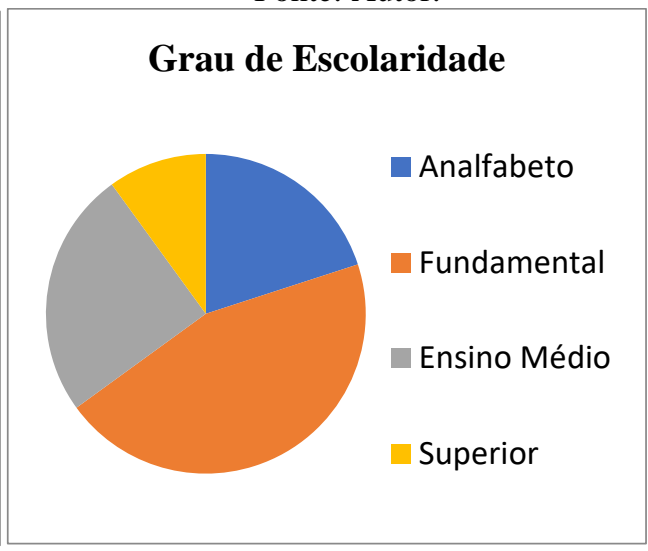

Diante dos gráficos expostos, pode-se compreender em um primeiro momento que a maioria dos clientes deste estabelecimento é do sexo feminino, enfatizando-se a ideia de que estas tendem a cuidar-se mais e posteriormente estão mais abertas a cuidar da sua saúde. Sendo necessário revelar também que o grau de escolaridade influencia muito na interação farmacêutico/cliente/paciente, visto que cada grau de instrução tende a elaboração de um plano de intervenção de programa de saúde diferente, como é o caso dos clientes da empresa, ou seja, explana-se que a grande maioria deste público alvo tem apenas o ensino fundamental ou nenhuma instrução, o que gera um maior cuidado na prestação de serviços farmacoterapicos. Sendo assim, vale salientar que, além disso, tem-se a renda variante como fator de influencia para aquisição de medicamentos, onde o farmacêutico tem um papel fundamental de apresentarlhes alternativas que possam proporcionar-lhe um tratamento eficiente, diante da existência de medicamentos genéricos e similares.Como pode-se observar ao longo destes dois meses, a adesão destes clientes do estabelecimentos a este serviço de atenção farmacêutica já pode melhorar cerca de 50\% dos hábitos diários dos clientes.

Visto que cerca de aproximadamente 400 pessoas passam mensalmente pela empresa para adquirir medicamentos no programa do Governo Federal “AQUI TEM FARMÁCIA POPULAR", sendo os medicamentos mais dispensados: 
Quadro 1: Medicamentos mais dispensados no programa Farmácia Popular

Fonte: Autor

\begin{tabular}{|cll|}
\hline Classe medicamentosa & \multicolumn{3}{c|}{ Medicamentos } \\
\hline Hipertensivos & Anlodipino, Captopril 25mg e \\
& Hidroclorotiazida 25mg & \\
\hline Antidiabetico & Metformina de 500 e 850 \\
& mg,Glibenclamida de $5 \mathrm{mg}$ e Insulina ( \\
& NPH e Regular); & \\
\hline Inibidor de Estatinas & Sinvastatina de 20 e 40 mg \\
\hline Tratamento de asma & Aerodini, Aerolin e Clenil \\
\hline Anticoncepcional & Ciclo 21 \\
\hline
\end{tabular}

Observa-se que este percentual de clientes ativos, tendiam a tomar incorretamente os medicamentos gerando a perca de medicamentos. Após aplicação da Atenção Farmacêutica e da assistência diária de farmacêutico, pode-se maximizar as boas práticas farmacêuticas diante da averiguação das posologias corretas e da adequação de horários corretos para uso destes medicamentos além de orientação de consultas frequentes com os médicos para análise das taxas e averiguação da necessidade de possíveis trocas de medicamentos pelos profissionais que faziam o acompanhamento e tratamentos destes clientes.

Deve-se explanar também que fora estes clientes, o estabelecimento ainda tem outros clientes ativos que fazem uso de outros medicamentos não fornecidos pelo governo e que sempre são auxiliados sobre a posologia correta de uso dos mesmos e das possíveis reações adversas, além de aferimento de pressões e orientações gerais de práticas básicas de saúde, gerando-se assim uma fidelização de todos os clientes diante da análise de suas necessidades básicas com serviços simples de assistência farmacêutica.

\section{c) Estratégias de implantação da Atenção Farmacêutica baseadas no método clínico de Correr e Otuki}

Diante de todos os dados ate então explorados, percebeu-se que o metido de Correr e Otuki (2013), deveria ser explanado para averiguação das boas praticas farmacêuticas, onde pode-se enxergar os seguintes dados diante das quatro etapas deste método: 
1) Acolher e Coletar dados do Paciente: neste item deve-se entender a necessidade de conhecimento dos clientes da organização diante do perfil de cada cliente, do histórico clinico e dos medicamentos ao qual iram fazer uso. Sendo assim, averiguou-se que o farmacêutico tem o cuidado de analisar cada caso individualmente através de conversas para entender como é a rotina do paciente/cliente, onde teve-se a observação de que em cada atendimento de atenção farmacêutica tem-se o cuidado de se perguntar quais as patologias que o paciente apresenta, se tem outros problemas de saúde fora os relativos aos medicamentos aos quais faz uso, e por fim saber se estes medicamentos apresentam alguma reação indesejada;

2) Identificar problemas relacionados á farmacoterapia: após aplicação do primeiro passo deste método, observou-se também que o farmacêutico tendo a fazer não só o acompanhamento inicial do público alvo desta pesquisa, como também uma análise continua. Além de identificar as possíveis advertências e informar aos pacientes quais os erros que estão cometendo durante o tratamento e o porquê de terem reagido negativamente aos medicamentos prescritos pelos médicos;

3) Elaborar um Plano de cuidado com o paciente:o farmacêuticoelabora metas de atendimento, que pode ser observado durante o aferimento de pressão dentre alguns pacientes, onde os mesmos fazer uso deste serviço através de um acompanhamento diário e anotações das possíveis irregularidades de sua P.A. (Pressão Arterial), tendo o intuito de assegurar que se forem observados casos excedentes fazer-se a intervenção de um tratamento através do aconselhamento de procura de um médico local para prescrição de outro medicamento para consequentemente ter eficácia do tratamento, projetando a análise médica através da carteirinha de acompanhamento individual da pressão.

4) Realizar o seguimento individual do paciente:e por fim, tem-se as relações de resultados e progressos nos casos de prestação de atenção farmacêutica. Onde verificou-se que as metas estabelecidas pelo farmacêutico são baseadas na prestação de informações farmacêuticas corretas para minimizar os efeitos da automedicação e do uso irregular de medicamentos, sendo necessário ressaltar que observou-se um progresso significativo de $50 \%$ de melhoria no uso de medicamentos através de auxilio nas posologias corretas de uso além de minimização de surgimento de novos problemas. 


\section{Considerações Finais}

A implantação do serviço de Atenção Farmacêutica é uma prática simples que tende a garantir tanto a fidelização dos clientes ativos como também dos possíveis clientes, sendo necessário que a organização enxergue do profissional farmacêutico um aliado para aquisição do custo beneficio entre lucro e maximização da saúde da população. Neste sentido percebese que a organização de estudo viu neste serviço uma oportunidade para garantir a fidelização dos clientes e garantir não só o lucro como também a satisfação de todos.

Sendo necessário se explanar que mesmo trazendo muitos benefícios a população, necessita-se constantes aperfeiçoamentos tanto do profissional farmacêutico como da organização para garantir cada vez mais adesões e motivação aos clientes usuários deste serviço para melhoria da qualidade de vida da população.

\section{Referências}

BOVO, F.; WISNIEWSKI, P.; MORKEI, M. L. L. Atenção Farmacêutica: Papel do Farmacêutico na Promoção de Saúde. Disponível em:<http://www.uel.br/ccb/patologia/portal/pages/arquivos/Biosaude\%20v\%2011\%202009/B S_v11_n1_DF_43.pdf $>$.

BRASIL. Ministério da Saúde. Portaria no 3.916, de 30 de outubro de 1998. Aprova a Política Nacional de Medicamentos. Diário Oficial da União, Poder Executivo, Brasília/DF, 10 nov. 1998. Disponível em: <bvsms.saude.gov.br/bvs/ saudelegis/gm/1998/prt3916_30_10_1998.html>.

Ministério da Saúde. Secretaria de Ciência, Tecnologia e Insumos Estratégicos. Departamento de AssistênciaFarmacêutica e Insumos Estratégicos. Assistência farmacêutica na atenção básica: instruções técnicas para sua organização / Ministério da Saúde, Secretaria de Ciência, Tecnologia e Insumos Estratégicos, Departamento de Assistência Farmacêutica e Insumos Estratégicos. - 2.ed. - Brasília : Ministério da Saúde, 2006.

CORRER, C. J. O serviço de clínicafarmacêutica. Brasil. Ministério da Saúde. Secretaria de Ciência, Tecnologia e Insumos Estratégicos. Departamento de Assistência

Farmacêutica e Insumos Estratégicos.Serviços farmacêuticos na atenção básica à saúde / Ministério da Saúde, Secretaria de Ciência, Tecnologia e InsumosEstratégicos. Departamento de Assistência Farmacêutica e Insumos Estratégicos. - Brasília : Ministério da Saúde, 2014. Disponível

em: 
<http://bvsms.saude.gov.br/bvs/publicacoes/servicos_farmaceuticos_atencao_basica_saude.pd f>.

DIÁRIO OFICIAL DA UNIÃO. AGÊNCIA NACIONAL DE VIGILÂNCIA SANITÁRIA. Disponível em: http://portal.anvisa.gov.br/documents/33880/2568070/RDC_44 _2009.pdf/ad27fafc-8cdb-4e4f-a6d8-5cc93515b49b.

GIL, Antônio Carlos. Como elaborar projetos de pesquisa. 4. ed. São Paulo: Atlas, 2002.

GÜNTHER, H. Pesquisa Qualitativa Versus Pesquisa Quantitativa: Esta É a Questão?. Disponível em: <http://www.scielo.br/pdf/\%0D/ptp/v22n2/a10v22n2.pdf>.

MESSIAS, M. C. F.; Atenção Farmacêutica no Uso Racional de Medicamentos. Disponível em:

$<$ http://arquivos.cruzeirodosuleducacional.edu.br/principal/new/revista_scienceinhealth/16_ja n_abr_2015/Science_06_01_07-14.pdf>.

MELO, D. V. A. Análise da importância do farmacêutico nas intervenções farmacêuticas. Disponível em:<http://www.ccecursos.com.br/img/resumos/ <farmacia/danielle-virginiaalmeida-melo-monografia-farmacia-hospitalar-clinica.pdf $>$.

NASCIMENTO, C. M; TOLEDO, J. Dificuldades de implantação da atenção farmacêutica e execução da seção I do capítulo VI da $\operatorname{RDC}^{\circ}$ 44, de 17 de agosto de 2009 em drogarias. Disponível em: <http://www.cpgls.pucgoias.edu.br/8mostra/Artigos/SAUDE\%20E\%20BIOLOGICAS/Dificu ldades\%20de $\% 20$ implanta $\% \mathrm{C} 3 \% \mathrm{~A} 7 \% \mathrm{C} 3 \% \mathrm{~A} 3 \mathrm{o} \% 20 \mathrm{da} \% 20$ aten $\% \mathrm{C} 3 \% \mathrm{~A} 7 \% \mathrm{C} 3 \% \mathrm{~A} 3 \mathrm{o} \% 20 \mathrm{far}$ mac\%C3\%AAutica\%20e\%20execu\%C3\%A7\%C3\%A3o\%20da\%20se\%C3\%A7\%C3\%A3o $\% 20 \mathrm{I} . \mathrm{pdf}>$.

REIS, A. M. M., Atenção Farmacêutica e Promoção do Uso Racional de Medicamentos. Disponível <http://www.ceatenf.ufc.br/Artigos/ATENFAR\%20e\%20URM\%20Adriano\%20Max.pdf>.

Como citar este artigo (Formato ABNT):

MATIAS, Aldemir Severo; MIRANDA, Thiago Adolfo Sobreira. Implantação do serviço de atenção farmacêutica em uma farmácia comunitária: um estudo exploratório na cidade de São José do Belmonte -PE. Id on Line Rev.Mult. Psic., 2018, vol.12, n.41, p.850-858. ISSN: 19811179 .

Recebido: 20/07/2018.

Aceito: $24 / 07 / 2018$ 